\title{
Fusion of duodeno-ileal omega switch and stomach pouch reconstruction as revisionary surgery for failed laparoscopic adjustable gastric banding and sleeved lesser curvature resection
}

\author{
Wojciech K. Karcz ${ }^{1}$, Cheng Zhou ${ }^{2}$, Piotr Małczak ${ }^{3}$, Hryhoriy Lapshyn ${ }^{4}$ \\ ${ }^{1}$ Department of Surgery, Division of Metabolic and Obesity Surgery, Schleswig-Holstein University Clinic, Campus Lübeck, Germany \\ ${ }^{2}$ Department of Hepatobiliary Surgery, Wuhan No. 1 Hospital, Wuhan, China \\ ${ }^{3} 2^{\text {nd }}$ Department of Surgery, Jagiellonian University Medical College, Krakow, Poland \\ ${ }^{4}$ Department of Surgery, Division Metabolic and Obesity Surgery, Schleswig-Holstein University Clinic, Campus Lübeck, Germany
}

Videosurgery Miniinv 2016; 11 (1): 49-53

DOI: $10.5114 /$ wiitm.2016.58336

\begin{abstract}
Revisionary bariatric surgery procedures are becoming more frequent. Facing the challenge of complicated digestive tract changes after redo surgery, it is crucial to choose an appropriate method. Duodeno-ileal omega switch (DIOS), with growing clinical value as an easy malabsorptive procedure, has emerged as a treatment option. We would like to present a case of a 52-year-old obese male patient with a history of laparoscopic adjustable gastric banding and removal of a lesser curvature as a revision procedure to restore the restriction. In order to improve the patient's health outcome, a two-stage re-revision procedure was performed, including DIOS to reduce the weight of the patient and a gastric pouch reconstruction in order to achieve a better satiety effect. This case study illustrates the intrinsic potential of malabsorption surgery and makes us believe that it may be used as the primary operation in two-staged procedures for revisionary surgical treatment.
\end{abstract}

Key words: sleeve gastrectomy, SADI-S, malabsorption, revisionary surgery, DIOS.

\section{Introduction}

Contrary to primary operations, revision bariatric procedures are heterogeneous and relatively complicated. In re-revisions, the technical demands are even more challenging. Generally, restrictive techniques such as revised sleeve gastrectomy (Re-SG) or malabsorptive procedures such as gastric bypass are used to improve health outcomes for patients with failing sleeve gastrectomies. However, in some cases multiple stomach manipulations make the Re-SG or bypass difficult to perform. Single-stage reoperations are potentially dangerous for morbidly obese patients with a history of failed bariatric surgery. Duodeno-ileal omega switch (DIOS) is charac- terized by a single omega anastomosis between the duodenum and ileum. The isolated biliopancreatic diversion (BPD) with Roux-en-Y was first introduced into bariatric surgery by Krawczykowski and presented several times at the International Federation for the Surgery of Obesity and Metabolic Disorders (IFSO) congresses. Torres modified BPD using the Traverso-Longmire duodeno-enterostomy reconstruction combined with SG, then our research group proposed a different length of the common channel to reduce the side effects $[1,2]$. After these modifications BPD became an alternative to biliopancreatic diversion with dudenal switch (BPD-DS) which is easier to perform and still leaves the option for a tra-

\section{Address for correspondence}

Piotr Małczak, $2^{\text {nd }}$ Department of Surgery, Jagiellonian University Medical College, 76b/70 Grzegórzecka St, 31-559 Krakow, Poland, phone: +48 601778 388, e-mail: pmmalczak@gmail.com 
ditional duodenal switch. All operations with pylorus preservation can be divided into the stomach and small intestine part by primary or revisionary surgery. Stage divided operations are gaining recognition among the surgical community and represent an interesting option especially for patients with a very high body mass index (BMI) $[2,3]$.

The aim of our study is to present our experience in treating patients with failed primary bariatric procedures and provide some insight into the methods we use at our clinic.

\section{Case report}

A 58-year-old male patient operated on with two bariatric procedures $(2005,2008)$ in another clinic visited us at the Lübeck University Hospital Outpatient Clinic in 2012 because of weight gain. In the history of the initial weight reduction surgery, he received an laparoscopic adjustable gastric banding (LAGB) performed in 2005, which was revised in 2008 with a lesser curvature SG performed without a clear reason instead of the greater curvature resection or Roux-en-Y gastric bypass (RYGB) (Figure 1). Probably technique selection was influenced by the following considerations: adhesions in gastric cardiac area, preserving blood supply in mentioned area and greater experience in esophageal resections than bariatric surgery. No improvement of body weight was achieved after this procedure. The patient's BMI prior to admission to our institute was $45 \mathrm{~kg} / \mathrm{m}^{2}$. Due to numerous comorbidities a twostage revision operation was planned in order to reduce perioperative risk associated with anamnestic reoperations, visceral adiposity and achieve a maximal effect with the least amount of intervention. The first stage was DIOS, which was performed for its malabsorptive component to reduce the weight and make the preparation of cardia adhesions easier in the second step. The second stage was a gastric pouch reconstruction for recovery of the restriction. It was performed 3 years and 4 months after the first stage.

\section{First stage: DIOS (Figure 2)}

The patient was placed in a lithotomy position with a reverse Trendelenburg tilt of $30^{\circ}$. The operator stood between the legs, which were set apart, with the first assistant on the left side of the patient.

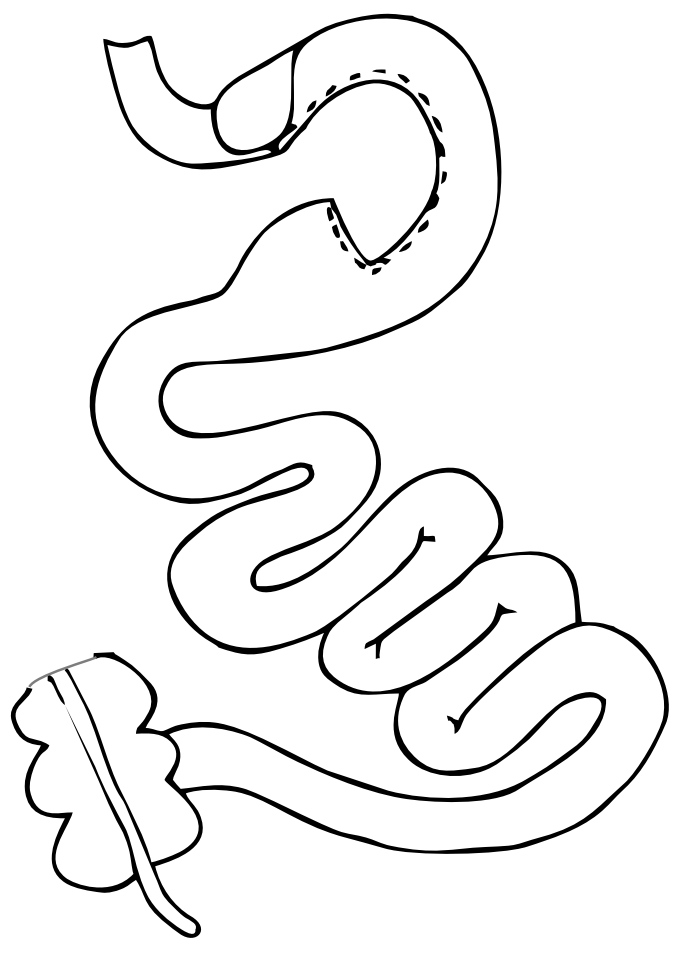

Figure 1. A stretched and narrow gastric sleeve after the lesser curvature has been removed (SG)

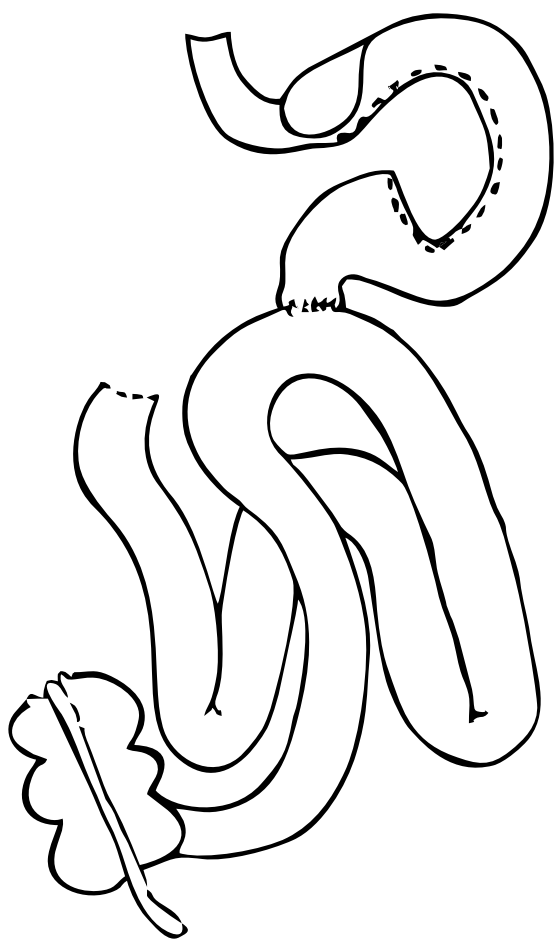

Figure 2. Duodeno-ileal omega switch (DIOS) 
Once pneumoperitoneum was achieved, the $\mathrm{CO}_{2}$ pressure was maintained at a level of $14 \mathrm{~mm} \mathrm{Hg}$. The camera port was placed by passing through the left rectus abdominis muscle. Exploration showed the stretched and narrow gastric sleeve with severe accumulation of visceral fat, which posed a risk for aggressive bariatric intervention. After preservation of the right gastric artery, the duodenum was divided by an Endo GIA stapler (Medtronic-Covidien, Dublin, Lila, $60 \mathrm{~mm}$ ). The length of the small intestine was measured laparoscopically from the Treitz ligament to the ileocecal valve. At $66 \%$ of the total length distal to the Treitz ligament, the ileum was brought to the post-pyloric duodenum and positioned in an ante-colic fashion [2, 3]. An E-hook was used to make two incisions parallel to the anastomosis: the first on the ileum and the second on the duodenum in a matching place. The duodeno-ileostomy was made in an end-to-side fashion by a hand-sewn running suture with 3/0 V-loc thread (Medtronic-Covidien, Dublin, Ireland). Two layers were established in the posterior wall of the anastomosis, and in the outer layer, the stapler line of the post-pyloric duodenum was included. Leakage testing of the anastomosis with methylene blue solution was performed, and when no leakages were seen the first stage, DIOS, was finished with placement of the drainage towards the duodenal stump.

\section{Second stage: gastric pouch restriction reconstruction (Figure 3)}

The patient's position and pneumoperitoneum were the same as described above for the DIOS maneuver. The second exploration clearly showed diminished amounts of visceral fat and liver volume, making the approach in the upper abdominal cavity easier (Photo 1). Because SG was done previously on the lesser curvature, peritoneal adhesions between visceral aspects of the liver and stomach were evident. After introducing a 32Ch gastric bougie, adhesiolysis was performed, and the gastrocolic and gastrosplenic ligaments were dissected from the greater curvature upstream until the fundus was fully exposed. Under the guidance of the gastric bougie, a pouch was created, similar to a gastric bypass pouch, using an Endo GIA (Medtronic-Covidien, Auto Suture) $60 \mathrm{~mm}$ stapler with Lila cartridges. Then, the pouch was brought to the distal gastric remnant and positioned (Photo 2). The outer layer of the posterior gastro-gastric anastomotic wall was subsequently

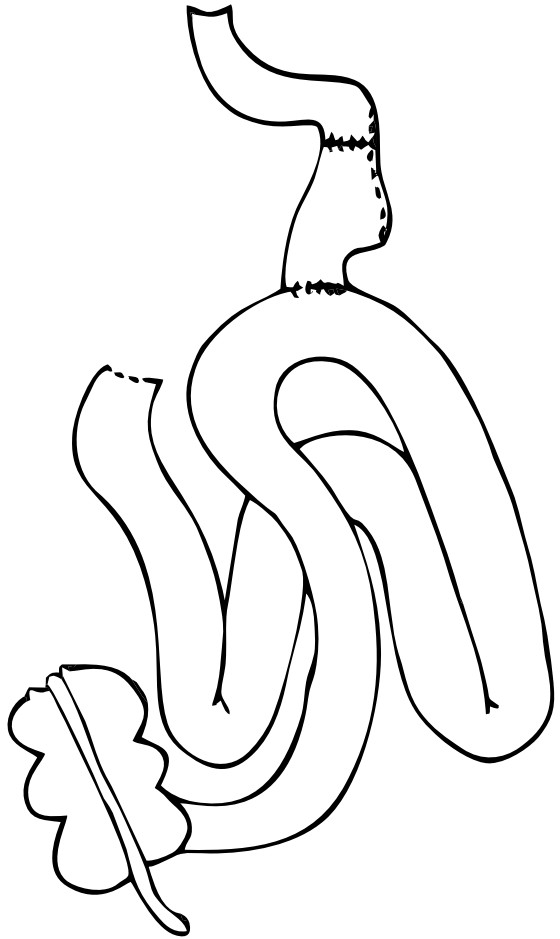

Figure 3. Reconstruction of the gastric passage

created with 3/0 V-loc (Medtronic-Covidien, Dublin, Ireland) in a hand-sewn pattern. The dissection of the greater curvature was continued until the cut was $6 \mathrm{~cm}$ upstream from the pylorus. Once redundant the omentum was removed. The remaining part of the gastric passage reconstruction anastomosis between the gastric pouch and the distal part of the gastric remnant was made with a hand sewing technique.

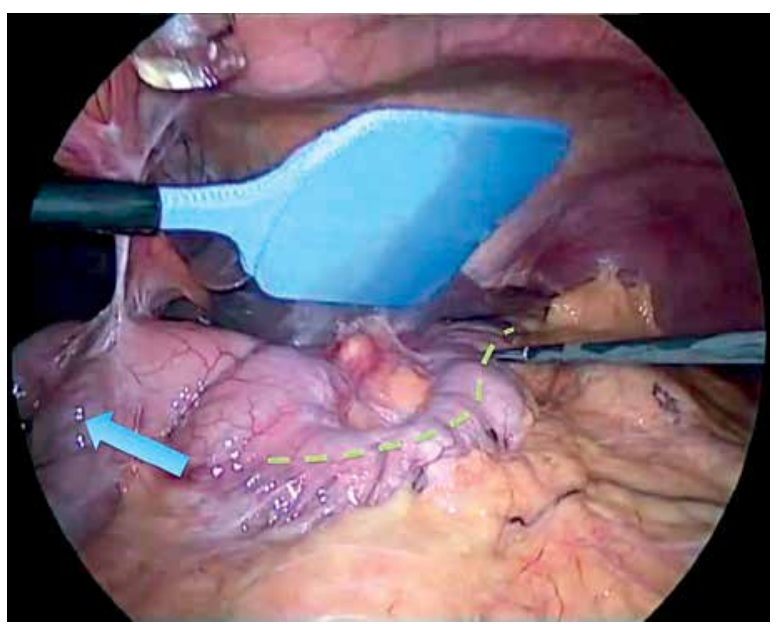

Photo 1. Blue arrow indicates the anastomosis of DIOS. Stretched gastric sleeve is indicated by the yellow dotted line 


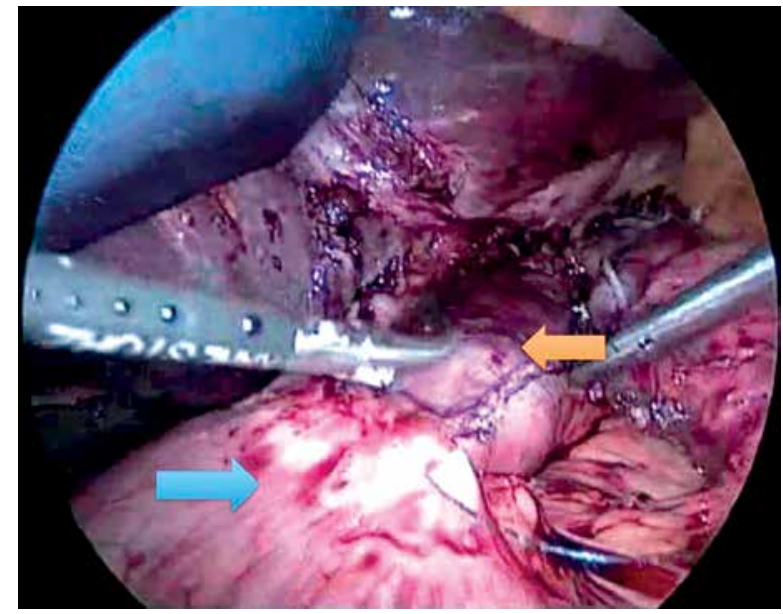

Photo 2. Distal part of the stomach (blue arrow) was approximated to the proximal gastric pouch (yellow arrow) during the reconstruction of the gastric passage

\section{Discussion}

The revision rate following bariatric surgery is about 2-20\% [4]. The most common reasons are insufficient weight loss and weight regain. Insufficient body weight loss is defined as a BMI greater than $40 \mathrm{~kg} / \mathrm{m}^{2} 2$ years after the primary operation. The definition of weight regain is a monthly body weight gain greater than $2 \mathrm{~kg}$ for 3 consecutive months. Nocca et al. estimated weight regain incidence after a sleeve gastrectomy as 2\% [5]. Furthermore, some researchers estimate the necessity for a reoperation after SG to be as high as 50\% [4]. Generally, when the gastric pouch presents as dilated, the control of body weight and correction of the metabolic disorder can be simply achieved by restoring the restriction of the gastrointestinal tract $[6,7]$. However, a combination of a vigorous restrictive and malabsorptive procedure is not always the first choice in such clinical situations. In our case, the patient received an unusual lesser curvature SG for the failure of LAGB and of course could not achieve the desired body weight reduction (Figure 1). The reason could be explained with reference to horizontal gastroplasty or the first gastric bypasses by Mason - both procedures led to large curvature and fundus dilatation, which provides a very small restrictive component [4]. This prompted us to perform a two-stage procedure: first to achieve a malabsorptive effect leading to weight loss and secondarily to restore the restrictive part of the stomach.
Sleeve gastrectomy is a restrictive procedure which first served as a component of BPD-DS [8, 9]. Normally, the postoperative volume of the gastric sleeve is set to $100-120 \mathrm{ml}$. Studies have shown that regain of body weight can occur if the primary gastric sleeve is greater than $200 \mathrm{ml}$ [5]. Our studies with 3D MSCT volumetry showed the size of the gastric sleeve to be $330 \mathrm{ml}$, which can be a reason for unsuccessful previous procedures, regain of weight by the patient and a need for a reoperation [10].

Roux-en-Y reconstruction is a widely used method in bariatric surgery for digestive tract reconnection during gastric bypass or duodenal switch. In 1967, the omega reconstruction according to Billroth 2 was first introduced in the bariatric field. Nowadays, Rutledge and his followers routinely apply it in mini gastric bypass [11]. Compared with Roux-en-Y it has the advantage of decreased complexity. The duodeno-jejunal omega switch (DJOS) and DIOS are even more physiologic because of the greater stomach pouch volume, avoiding biliary reflux and histopathological changes in the stomach mucosa [2]. In our case, the small curvature side of the stomach was managed surgically twice (once for a biopsy and once for the lesser curvature gastric sleeve). To avoid complications due to adhesions, scar formation, and poor blood supply in addition to severe accumulation of visceral fat, which posed a large risk for repeated manipulations of the stomach, it is important to limit surgical interventions to a minimum, giving the patient the best possible weight reduction and metabolic outcome. Six months after DIOS, the patient had lost $40 \mathrm{~kg}$, and his physical condition and visceral fat and diabetes status were consequently improving. The outcome proved that leaving the gastric intervention for a second phase was a wise choice. We believe that DIOS not only allows one to avoid the dangers of larger surgery on the stomach due to high BMI, but also makes it possible to decrease the body weight during the first stage because of malabsorption, which is the determining factor for the success of restoration of the gastric pouch restriction in the second phase. Currently, to minimize the risks related to morbidly obese patients, adopting a restrictive procedure as a first stage operation followed by a malabsorptive operation to enhance the weight control is universally acknowledged [12, 13]. This case shows that choosing DIOS as an option to fulfill the aim of multi-step bariatric surgical therapy can be a treatment leading to significant 
health improvement in complicated patients requiring a reoperation.

However, as in the classic B2 gastrectomy, the biliary reflux and dumping syndrome are two of the common complications. The idea of the preservation of the pylorus in DIOS derives from pylorus-preserving pancreaticoduodenectomy (PPPD). The intact pylorus can effectively protect the small intestine from the excessive gastric acid, and prevent biliary reflux and dumping as well. Performing an omega reconstruction between the duodenum and ileum, the length of the common channel (CC) was tailored to one third of the total length of the small bowel. Because variation exists among individual small intestines, the lengths of the common channel vary. Thus, laparoscopic measurement from the Treitz ligament to the ileocecal valve should always be performed. In our case, the CC was $280 \mathrm{~cm}$, which in principal should not be shorter than $200 \mathrm{~cm}$ [14]. In contrast to DJOS with a CC as long as 2/3 of the small intestine length, a shorter CC in DIOS strengthens the malabsorptive component undoubtedly, but the optimal length for the common channel needs to be further evaluated in randomized and prospective trials [2, 3].

All modern bariatric operations consist of a gastric restrictive component, such as a small (banded/ not banded) gastric pouch, (banded/not banded) sleeve, stomach volume reduction by BPD procedure or a vertical banded gastroplasty (VBG). Only with the restoration of the gastric restriction and sufficient mechanical limitation can neural satiety be properly achieved. Therefore, the reconstruction of the gastric passage as a second stage procedure is essential to subsequent improvement of body weight and metabolic disorders. For this reason, we removed the stretched part of the sleeve in the middle/upper part, and then reconnected the well-perfused, scarless distal part with the proximal stomach. At the 1-year follow-up the BMI of the patient was $26 \mathrm{~kg} / \mathrm{m}^{2}$.

\section{Conclusions}

In this case, we delicately employed a two-stage operation, DIOS and reconstruction of the gastric pouch, and successfully corrected a rare, dysfunctional, lesser curvature resection by lesser curvature sleeve gastrectomy after LAGB. Experience in various bariatric procedures allowed us to devise a technique that combined the advantages of these procedures, which enabled us to perform a successful revision surgery also in this case.

\section{Conflict of interest}

The authors declare no conflict of interest.

\section{References}

1. Sánchez-Pernaute A, Herrera MA, Pérez-Aguirre ME, et al. Single anastomosis duodeno-ileal bypass with sleeve gastrectomy (SADI-S). One to three-year follow-up. Obes Surg 2010; 20: 1720-6.

2. Karcz KW, Karcz-Socha I, Kuesters S, et al. Duodeno-entero omega switch with gastric plication - more physiological technique in metabolic surgery. Videosurgery Miniinv 2013; 8: 273-9.

3. Grueneberger JM, Karcz-Socha I, Marjanovic G, et al. Pylorus preserving loop duodeno-enterostomy with sleeve gastrectomy - preliminary results. BMC Surg 2014; 14: 20.

4. Karcz WK, et al. Principals of metabolic revisional burgery. In: Principals of metabolic surgery. Karcz WK, Thomusch O (eds). Springer, Berlin Heidelberg 2012; 349-66.

5. Nocca D, Krawczykowsky D, Bomans B, et al. A prospective multicenter study of 163 sleeve gastrectomies: results at 1 and 2 years. Obes Surg 2008; 18: 560-5.

6. Langer FB, Bohdjalian A, Felberbauer FX, et al. Does gastric dilatation limit the success of sleeve gastrectomy as a sole operation for morbid obesity? Obes Surg 2006; 16: 166-71.

7. DeMaria EJ, Sugerman HJ, Meador JG, et al. High failure rate after laparoscopic adjustable silicone gastric banding for treatment of morbid obesity. Ann Surg 2001; 233: 809-18.

8. Johnston D, Dachtler J, Sue-Ling HM, et al. The Magenstrasse and Mill operation for morbid obesity. Obes Surg 2003; 13: 10-6.

9. Weiner RA, Weiner S, Pomhoff I, et al. Laparoscopic sleeve gastrectomy - influence of sleeve size and resected gastric volume. Obes Surg 2007; 17: 1297-305.

10. Karcz WK, Kuesters S, Marjanovic G, et al. 3D-MSCT gastric pouch volumetry in bariatric surgery - preliminary clinical results. Obes Surg 2009; 19: 508-16.

11. Rutledge R. The mini-gastric bypass: experience with the first 1,274 cases. Obes Surg 2001; 11: 276-80.

12. Silecchia G, Boru C, Pecchia A, et al. Effectiveness of laparoscopic sleeve gastrectomy (first stage of biliopancreatic diversion with duodenal switch) on co-morbidities in super-obese high-risk patients. Obes Surg 2006; 16: 1138-44.

13. Baltasar A, Serra C, Pérez N, et al. Laparoscopic sleeve gastrectomy: a multi-purpose bariatric operation. Obes Surg 2005; 15 : 1124-8.

14. Sánchez-Pernaute A, Rubio MÁ, Pérez Aguirre E, et al. Single-anastomosis duodenoileal bypass with sleeve gastrectomy: metabolic improvement and weight loss in first $100 \mathrm{pa}$ tients. Surg Obes Relat Dis 2013; 9: 731-5.

Received: 16.09.2015, accepted: 19.01.2016. 